\title{
Información para pacientes y educación en la salud de la ciudadanía: a la búsqueda de nuevos usuarios
}

\author{
Por Aurora Vall y Concepción Rodríguez-Parada
}

\begin{abstract}
Resumen: La elaboración de un marco legal y normativo que fija el derecho de los ciudadanos a una información veraz y adecuada sobre salud y enfermedad implica tener en cuenta estas necesidades informativas desde la doble perspectiva del "consumer health" y el "patient education". Se defiende, desde el punto de vista de los profesionales de la información, la materialización de este derecho a la información y a la formación en temas relacionados con la salud y la enfermedad gracias a un mejor aprovechamiento de los recursos informativos disponibles en las bibliotecas médicas y a los profesionales que los gestionan.

Palabras clave: Bibliotecas para pacientes, Bibliotecas públicas, Consumer health, Derecho a la información, Hospitales, Humanización de la asistencia sanitaria, Patient education.
\end{abstract}

Title: Patient education and consumer health: searching for new users

Abstract: The development of a legal and regulatory framework to establish the right of citizens to accurate and sufficient information about their own illnesses and health education requires that their information needs be considered from the double perspective of patient education and consumer health. The objective of this article is to show the contribution of librarians in converting to reality this right, based on informational literacy in medical and health information resulting from the optimal use of information resources available in public and medical libraries and the expertise of library professionals.

Keywords: Libraries for patients, Public libraries, Consumer health, Freedom of information, Hospitals, Humanization of assistance, Patient education.

Vall, Aurora; Rodríguez-Parada, Concepción. "Información para pacientes y educación en la salud de la ciudadanía: a la búsqueda de nuevos usuarios". El profesional de la información, 2010, mayo-junio, v. 19, n. 3, pp. $296-299$.

DOI: 10.3145/epi.2010.may.11

\section{Introducción}

TRADICIONALMENTE SE HA ASOCIADO la información medico-sanitaria a los profesionales de la salud y disciplinas afines. Emerge sin embargo un colectivo de usuarios al que hasta ahora se ha prestado una pobre atención: los ciudadanos. Éstos necesitan también tener garantizado el acceso a la información sobre la salud y la enfermedad porque en última instancia ambas son parte intrínseca de su existencia.
Desde hace algunos años muchos países del primer mundo están elaborando un marco legal y normativo que reconoce el derecho de los ciudadanos a una información veraz y adecuada sobre salud y enfermedad ${ }^{1}$.

Hasta ahora los distintos proyectos que se han puesto en marcha se dirigían al paciente y eran en su mayoría fruto de actuaciones extrahospitalarias: asociaciones de voluntarios de variado signo, bibliotecas públicas y otras. La nueva legislación introduce cambios cuyas consecuencias implican modificar en algunos aspectos sustanciales la relación médico-paciente a la vez que entran en juego otros elementos provenientes del propio centro hospitalario. Un hospital debe proporcionar a la población una asistencia medico-sanitaria completa, tanto curativa como preventiva, siendo además, en muchos casos, un centro de investigación y de formación del personal sanitario.

El hospital debe velar simultáneamente por llevar a cabo una gestión basada en criterios de efi- 
cacia, eficiencia y equidad. En un contexto de búsqueda y demanda de calidad asistencial, el hospital debe satisfacer las necesidades de los enfermos tanto desde una perspectiva científica (conocimientos, diagnóstico, tecnología y terapéutica) como humana, en lo que se suele denominar "humanización de la asistencia", que incluye entre otros el trato al enfermo y sus familiares y el proporcionarles información correcta y adecuada (Asenjo-Sebastián, 2006).

Uno de los indicadores de evaluación en los procesos de acreditación de los hospitales es la promoción del principio de autonomía del paciente; es decir, si el centro médico facilita que el paciente $\mathrm{y}$ su entorno participen en el proceso de toma de decisiones sobre su asistencia, para lo cual obviamente el suministro de información es cla$\mathrm{ve}^{2}$.

\section{"El ciudadano tiene derecho a una información veraz y adecuada sobre salud y enfermedad"}

Por tanto, la oferta de servicios informativos sobre aspectos de salud y enfermedad se plantea hoy gracias a la feliz coincidencia de tres factores, algunos de ellos ya apuntados: el primero, la percepción del derecho a la información como un derecho humano más; el segundo, la búsqueda de un modelo de gestión hospitalaria basado en criterios de calidad; y el último, el reconocimiento (parcial a veces) de la función mediadora del bibliotecario $^{3}$.

El propósito del presente artículo es mostrar cómo los bibliotecarios podemos contribuir a la materialización del derecho a la información y a la formación (concebida como alfabetización informacional) en aspectos relacionados con la salud y la enfermedad, gracias a un uso óptimo de los recursos informativos disponibles en las bibliotecas (médicas y públicas) y a un aprovechamiento integral de las competencias de sus profesionales.

\section{Una propuesta viable}

Debe partir de las necesidades informativas del ciudadano-usuario concebido desde una doble perspectiva: el ciudadano enfermo (y/o familiares y acompañantes) necesitado de información sobre su propia enfermedad (patient education), y el ciudadano sano necesitado de información sobre la salud para prevenir la enfermedad (consumer health).

En ambos casos los contenidos informativos deben tener su origen en una biblioteca especializada que garantice su calidad científica y la adecuación a la demanda. Ahora bien, la naturaleza de esta información y los canales para tener acceso a ella variarán en cada caso. Sea cual sea el procedimiento final seguido, se tiene que partir de la base que bibliotecarios y médicos deben colaborar activamente a la hora de resolver las necesidades de información ${ }^{4}$.

\subsection{Informar y educar a los ciu- dadanos enfermos}

En estos casos, y centrándonos en el contexto hospitalario, la secuencia del proceso debería ser la siguiente: el médico informa primero a los pacientes (y/o familiares) sobre el diagnóstico, pronóstico y tratamiento, tal y como se explicita en su código deontológico profesional; a continuación, a demanda del usuario y con la supervisión del equipo médico, el bibliotecario continúa el proceso informativo (Caphis, 1996).

Para que esto sea posible debe potenciarse la figura del médico divulgador científico, es decir, del profesional que elabora información sobre su especialidad teniendo en cuenta que los receptores van a ser ciudadanos con perfiles muy diversos. En este sentido, se deberán tener en cuenta muchas variables en relación con los usuarios potenciales, tales como el nivel educativo (desde una escolarización no finalizada hasta una formación superior), la procedencia geográfica (no sólo por razón del idioma sino también cultural y antropológica) o el tipo de información necesaria (varia según la naturaleza de la enfermedad pero también según el estadio en que se encuentra el paciente), entre otras.

En este contexto el bibliotecario se convierte en el canal informativo por excelencia, a partir de su triple función, siempre avalada por su código deontológico:

- elaborar productos informativos (a partir de la información primaria de los médicos así como de su colaboración y asesoramiento en la revisión de los productos finales),

- diseñar servicios, y

- realizar la difusión adecuada de todo ello ${ }^{5}$.

Con esta propuesta se pretende rentabilizar los recursos de muchos hospitales tanto desde el punto de vista de la información como de los profesionales que allí trabajan y conviven. Así, la información adquirida para uso exclusivo de los profesionales medico-sanitarios tiene un nuevo uso y, por lo tanto, su coste se verá nuevamente amortizado; a los profesionales, por su parte, se les pide que realicen aquello que mejor sabe hacer cada uno y que forma parte intrínseca de sus funciones.

Para que esta propuesta sea viable es imprescindible que el hospital, concebido como un todo, se implique, que la dirección del centro comprenda la importancia del proceso informativo y formativo de 
los pacientes y lo asuma como un objetivo prioritario. En el contexto hospitalario, la biblioteca para pacientes puede y debe cooperar en este proceso informativo y educativo (IFLA, 2001).

En este sentido, desde hace ya algunos años la Oficina Regional para Europa de la OMS promueve el proyecto Health Promotion in Hospitals $(\mathrm{HPH})^{6}$. Un hospital promotor de la salud, además de las actividades curativas y rehabilitadoras que le son propias, participa activamente en la mejora de la promoción de la salud de la población mediante dos vías de actuación: las acciones orientadas a la prevención de las enfermedades, sobre todo las crónicas, y la educación en la salud. Las principales líneas de trabajo que se están llevando a cabo son las mejoras en la comunicación con los pacientes y el establecimiento de estrategias de promoción de la salud ${ }^{7}$.

En las directrices publicadas para la implementación de dicho proceso (WHO/ROE, 2004), a pesar de explicitarse la necesaria colaboración del hospital con otras instituciones, servicios y entidades, y la optimización de recursos relacionados con la información sobre la salud y la enfermedad, no se hace ninguna alusión al gran potencial informativo y humano que representan las bibliotecas médicas y sus profesionales.

\subsection{Educar en la salud y los hábi- tos saludables a los ciudadanos}

La propuesta anterior, a pesar de tener en cuenta la educación en la salud, se centra en el ciudadano enfermo y, sobre todo, en el hecho de que la información se crea, gestiona y difunde directamente en el hospital. Sin embargo es necesario avanzar y ampliar tanto el radio de actuación como los recursos a utilizar, siendo uno de ellos la biblioteca pública.

Ésta es una infraestructura ya existente y su implicación en la educación en la salud forma parte de sus funciones. Según el Manifiesto de la Unesco sobre la biblioteca pública (1994), ésta "constituye un requisito básico para el aprendizaje a lo largo de los años, para la toma independiente de decisiones y el progreso cultural del individuo $y$ los grupos sociales". Se trata pues de dar cabida de una manera más evidente a un nuevo servicio en el marco de su funcionamiento habitual.

Hay una serie de factores contextuales que favorecen esta cooperación entre hospital y biblioteca pública. Entre ellos cabe destacar: el aumento de enfermedades crónicas y el progresivo envejecimiento de la población, así como el incipiente movimiento ciudadano que reclama una información de calidad en aspectos relacionados con la salud; existe además, sin obviar los aspectos éticos y de derechos humanos, un interés económico. Se ha demostrado que una educación sanitaria generalizada permite reducir las visitas médicas y el gasto farmacéutico. Las consecuencias son claras: los costes sanitarios que debería asumir la administración disminuirían sensiblemente (Roth, 1978).

\section{"La biblioteca pública y las bibliotecas especializadas en medicina deben cooperar activamente en la tarea de educar en la salud"}

La biblioteca pública, en estrecha colaboración con las bibliotecas especializadas en medicina y los profesionales de la salud, puede convertirse en centro educador, informador y difusor abierto al conjunto de la ciudadanía. Estas actuaciones pueden concretarse en servicios de muy diversa naturaleza, adaptados a cada realidad social concreta: desde las conferencias y debates realizados dentro de los muros de la biblioteca (y no del hospital como hemos expuesto en el caso anterior) por profesionales, a la presentación de asociaciones, la elaboración de productos concretos como el acceso en línea a recursos sobre salud y sanidad previamente validados por especialistas, la difusión de trípticos informativos o la revisión de la colección médica, pero también el aprendizaje del uso de las tecnologías para el acceso a información de calidad sobre salud, entre muchos otros. La biblioteca pública se convierte en mediadora y pone sus instalaciones, su colección, sus servicios bibliotecarios y sus profesionales al servicio de la información sobre la salud.

En España ${ }^{8}$ se trata de una propuesta relativamente novedosa, pero en otros países hay destacados antecedentes. Es remarcable el proyecto impulsado desde la última década del siglo XX por el National Health System (NHS) del Reino Unido que propone una aproximación entre la biblioteca pública y la biblioteca especializada en medicina. Se trata de proporcionar por parte de la biblioteca pública servicios específicos de información sobre la salud, generalmente recursos previamente seleccionados por especialistas que garantizan la calidad y la adecuación de los contenidos al perfil de los usuarios de la biblioteca pública; pero también puede producirse un movimiento de usuarios desde la biblioteca pública a la especializada cuando las demandas de información superen los recursos propios.

\section{Conclusiones}

La propuesta aquí esbozada pretende aunar esfuerzos de los distintos colectivos que intervienen en el proceso informativo y formativo del ciudadano en relación con la enfermedad y la salud, partiendo del 
uso de recursos ya existentes. Pretendemos además reivindicar la figura del profesional de la información como agente imprescindible en este proceso.

\section{"Es imprescindible que médicos y bibliotecarios colaboren estrechamente"}

Se trata de una propuesta ambiciosa porque implica la intervención de distintos colectivos con perfiles distintos tanto en la formación como en la manera de trabajar. Significa también la colaboración de distintas instituciones y administraciones públicas en muchos casos. Además carecemos de tradición en este tipo de proyectos transversales y conceptuales. Pero a pesar de ello y teniendo en cuenta la evolución informativa de la sociedad actual así como la tendencia generalizada a una racionalización de los recursos, los hospitales y los estamentos sanitarios en general deberían considerar esta nueva formulación del proceso informativo y formativo de los pacientes.

No puede hacerse caso omiso a esta necesidad de información de los ciudadanos en relación con la salud y la enfermedad ya que si los responsables (los hospitales, la administración) no la procuran, no significa que los usuarios cejen en su empeño y consigan información que no siempre cumpla con las mínimas garantías de calidad y adecuación. Las TIC facilitan el acceso a cualquier información no controlada y en cuestiones tan sensibles como la salud y la enfermedad, la intervención de profesionales es fundamental. Si los ciudadanos van a acceder a la información deseada en cualquier circunstancia, es preferible establecer previamente procedimientos, crear colecciones y servicios adecuados y garantizar así el resultado final: una información de calidad, educación en la salud y aprendizaje del uso de las TIC, esencial en estos procesos informativos y formativos.

\section{Notas}

1. En trabajos anteriores hemos presentado una detallada relación de las leyes y normas que configuran este marco legal y normativo. Destacamos aquí la Llei 21/2000, de 29 de desembre sobre els drets d'informació concernent a la salut i l'autonomia del pacient, pionera en España; la Declaración para la promoción de los derechos de los pacientes en Europa (1994) y la Ottawa charter for health promotion, 1986 (2000), ambas de la WHO/EURO; el Manifiesto de IFLA Unesco sobre la biblioteca pública, 1994 y las Pautas para bibliotecas al servicio de pacientes de hospital (2001) de la IFLA, así como los códigos deontológicos profesionales de médicos y bibliotecarios, entre otros.

2. En el caso de los hospitales catalanes, por ejemplo, este aspecto queda recogido en el "Criteri 5 Processos. Subcriteri 5.b Producció, distribució servei d'atenció dels productes i serveis. Ètica drets del client", y en el "Criteri 6 Resultats en els clients. Subcriteri 6.a Mesures de percepció". Para una información más detallada, véase: Acreditació de centres d'atenció hospitalària aguda a Catalunya. Barcelona: Generalitat de Catalunya. Departament de Salut, 2005. 2 vol.

3. Sin embargo, este panorama es incompleto si no se alude al código deontológico de los médicos que reconoce explícitamente el derecho a la información de los pacientes aunque se atribuyen la responsabilidad exclusiva de ofrecerla.

4. Para más información sobre la colaboración entre profesionales, bibliotecarios y médicos desde una perspectiva legal y deontológica, véase: Vall, Aurora; Rodríguez-Parada, Concepción "El derecho a la información del paciente: una aproximación legal y deontológica". BiD: textos universitaris de biblioteconomia i documentació, 2008, desembre, n. 21.

http://www.ub.edu/bid/21/vall2.htm

5. Para un ejemplo de buenas prácticas en el que colaboran médicos, bibliotecarios, pacientes y familiares, véase: Monroe Carell Jr Children's Hospital at Vanderbilt. Health library.

http://www.vanderbiltchildrens.org/interior.php? mid $=996$

6. Véase: WHO/ROE. Health promoting hospitals. http://www.euro.who.int/healthpromohosp

7. El Hospital Clínic de Barcelona, miembro de la red, es un ejemplo de buenas prácticas con dos proyectos emblemáticos: Forumclínic: programa interactivo para pacientes y Fórum Salut Clínic http://www.forumclinic.org/?set_language $=e s \&$ $c l=e s$

http://www.hospitalclinic.org/Secciones/Ciuda dano/Educacionensalud/F\%C3\%B2rumSalu tCl\%C3\%ADnic/tabid/928/language/es-ES/ Default.aspx.
8. Un tímido intento es el que se realiza desde la Biblioteca pública de Can Peixauet de Santa Coloma de Gramanet (Barcelona): la proximidad física entre la biblioteca y el hospital local de referencia (Hospital de l'Esperit Sant) así como la buena sintonía entre los profesionales de ambas instituciones ha propiciado que en la biblioteca se celebren conferencias periódicas sobre temas relacionados con la salud.

\section{Bibliografía}

Asenjo-Sebastián, Miguel-Ángel. "Fundamentos de planificación y gestión hospitalarias". En: Gestión diaria del hospital. Asenjo-Sebastián, Miguel-Ángel (dir.). $3^{\mathrm{a}}$ ed. Barcelona: Masson, 2006, pp. 15-46. ISBN 84-458-1666-7.

Caphis. "The librarian's role in the provision of consumer health information and patient education". Bulletin of the Medical Libraries Association, 1996, April, v. 84, n. 2, pp. 238-239.

http://caphis.mlanet.org/resources/caphis_state ment.html

IFLA. Pautas para bibliotecas al servicio de pacientes de hospital, ancianos y discapacitados en centros de atención de larga duración. Nancy M. Panella, dir. The Hague, 2001, ISBN 9070916819.

http://www.ifla.org/VII/s9/nd1/iflapr-69s.pdf

Rodríguez-Parada, Concepción; Vall, Aurora. "El derecho a la información del enfermo hospitalizado". Boletín de la Anabad, 2006, v. LVI, n. 3, pp. 165-185. ISSN 0210-4164.

Roth, Britain G. "Health information for patients: the hospital library's role". Bulletin of Medical Library Association, 1978, January, v. 66, n. 1, pp. 14-18.

http://ukpmc.ac.uk/picrender.cgi? artid=344368 \&blobtype $=p d f$

Vall, Aurora; Rodríguez-Parada, Concepción. "El dret a la informació dels pacients: metges i bibliotecaris, un camí per recórrer plegats". Annals de medicina, 2009, juliol/agost/setembre, v. 92, n. 3, pp. 104-106.

http://webs.academia.cat/pages/academ/vidaa cad/publica/annals/2009/A3/vim2.htm

WHO/ROE. Standards for health promotion in hospitals, 2004.

http://www.euro.who.int/document/e82490.pdf

WHOIROE. The international network of health promoting hospitals and health services: integrating health promotion into hospitals and health services: concept, framework and organization, 2007.

http://www.euro.who.int/document/E90777.pdf

Aurora Vall, Concepción Rodríguez-Parada, Facultat de Biblioteconomia i Documentació, Universitat de Barcelona.

Melcior de Palau, 140.

08014 Barcelona

auroravall@ub.edu

crodriguezp@ub.edu 